\title{
Discovery of magnetic fields in three He variable Bp stars with He and Si spots ${ }^{\star}$
}

\author{
M. Briquet ${ }^{1, \star \star, \circ}$, S. Hubrig ${ }^{2}$, M. Schöller ${ }^{2}$, and P. De Cat $^{3}$ \\ 1 Instituut voor Sterrenkunde, Katholieke Universiteit Leuven, Celestijnenlaan 200 B, B-3001 Leuven, Belgium \\ 2 European Southern Observatory, Casilla 19001, Santiago 19, Chile \\ 3 Koninklijke Sterrenwacht van België, Ringlaan 3, B-1180 Brussel, Belgium
}

The dates of receipt and acceptance should be inserted later

\begin{abstract}
Key words stars: chemically peculiar - stars: individual (HD 55522, HD 105382, HD 131120, HD 138769) - stars: magnetic fields

It is essential for the understanding of stellar structure models of high mass stars to explain why constant stars, nonpulsating chemically peculiar hot Bp stars and pulsating stars co-exist in the slowly pulsating B stars and $\beta$ Cephei instability strips. We have conducted a search for magnetic fields in the four Bp stars HD 55522, HD 105382, HD 131120, and HD 138769 which previously have been wrongly identified as slowly pulsating B stars. A recent study of these stars using the Doppler Imaging technique revealed that the elements He and Si are inhomogeneously distributed on the stellar surface, causing the periodic variability. Using FORS 1 in spectropolarimetric mode at the VLT, we have acquired circular polarisation spectra to test the presence of a magnetic field in these stars. A variable magnetic field is clearly detected in HD 55522 and HD 105382, but no evidence for the existence of a magnetic field was found in HD 131120. The presence of a magnetic field in HD 138769 is suggested by one measurement at $3 \sigma$ level. We discuss the occurence of magnetic B stars among the confirmed pulsating B stars and find strong magnetic fields of order $\mathrm{kG}$ and oscillations to be mutually exclusive.
\end{abstract}

(C) 2006 WILEY-VCH Verlag GmbH \& Co. KGaA, Weinheim

\section{Introduction}

In the framework of a long-term monitoring project dedicated to the study of seismic models for a large sample of slowly pulsating B (SPB) stars (Aerts et al. 1999, Mathias et al. 2001) four variable B-type stars - HD 55522, HD 105382, HD 131120, and HD 138769 - have been identified as chemically peculiar $\mathrm{He}$-variable stars (Briquet et al. 2004). All available data sets revealed that these stars are monoperiodic variables. A comparison of moment variations of silicon and helium lines allowed to conclude that the observed variability of these four stars must be attributed to an inhomogeneous distribution of chemical elements on the stellar surface. All four stars are moderate rotators with $v \sin i \in[70,86] \mathrm{km} / \mathrm{s}$. Using high resolution CAT/CES spectra, Briquet et al. (2004) produced abundance maps for both silicon and helium on the stellar surface using the Doppler Imaging technique. Currently, these maps are the only existing Doppler images generated for hot B-type stars with an effective temperature as high as $18,000 \mathrm{~K}$.

All previous studies of chemically peculiar Ap and Bp stars with inhomogeneous distributions of elements on the stellar surface show that these stars are also known to have variable magnetic fields, generally diagnosed through mean

\footnotetext{
$\star$ Based on observations collected at the European Southern Observatory, Paranal, Chile (ESO programmes 71.D-0308(A), 072.D-0377(A), and 073.D-0466(A)).

$\star \star$ Postdoctoral fellow of the Fund for Scientific Research, Flanders.

○Corresponding author: e-mail: maryline@ster.kuleuven.be
}

longitudinal magnetic field, mean magnetic field modulus or net broadband linear polarisation measurements. When these observations are interpreted within the framework of the oblique rotator model, we obtain a self-consistent picture of a star whose atmosphere contains a heterogeneous distribution of chemical elements, and is permeated by a static, quasi-dipolar magnetic field. As the star rotates, we observe the magnetic field and surface abundance distribution from various aspects, resulting in variability of the measured magnetic field and line strength. The peculiarities in the Ap and Bp stars are believed to result from an upward selective diffusion of some elements, for which the radiative force exceeds the downwards gravitational settling of other elements. Such a separation produces apparent overabundances in the floating elements and underabundances of the sinking ones in the atmospheres of chemically peculiar stars (e.g., Michaud 1970; Michaud et al. 1981).

Four magnetic field observations have been obtained by Borra et al. (1983) for HD 131120 in 1981. However, they showed no sign of the presence of a magnetic field. No measurements of magnetic fields for HD 55522, HD 105382, and HD 138769 have been reported in the literature. In this paper, we present the first magnetic observations of all four stars which we gathered with FORS 1 at the VLT in the last years and discuss the magnetic data in view of the $\mathrm{He}$ and Si distributions derived in Briquet et al. (2004). 
Table 1 The mean longitudinal field measurements for our sample of Bp stars observed with FORS 1 in the frame of our ESO service programs 71.D-0308, 072.D-0377, and 073.D-0466. In the first four columns we give the HD number, another identifier, the $V$ magnitude and the spectral type. In Columns 5 and 6 we present the modified Julian date of the middle of the exposures and the corresponding measured mean longitudinal magnetic field $\left\langle B_{1}\right\rangle$. For each star we give the rms longitudinal magnetic field and the reduced $\chi^{2}$ for all measurements in Columns 7 and 8.

\begin{tabular}{|c|c|c|c|c|c|c|c|}
\hline HD & $\begin{array}{c}\text { Other } \\
\text { Identifier }\end{array}$ & $V$ & Sp. Type & MJD & $\begin{array}{l}\left\langle B_{1}\right\rangle \\
{[\mathrm{G}]}\end{array}$ & $\begin{array}{l}\left\langle\overline{\left\langle B_{l}\right\rangle}\right. \\
{[\mathrm{G}]}\end{array}$ & $\chi^{2} / n$ \\
\hline \multirow[t]{4}{*}{55522} & HR2718 & 5.9 & B2IV/V & 52999.190 & $38 \pm 73$ & 518 & 65.1 \\
\hline & & & & 52999.227 & $39 \pm 234$ & & \\
\hline & & & & 53000.053 & $873 \pm 66$ & & \\
\hline & & & & 53275.295 & $554 \pm 60$ & & \\
\hline \multirow[t]{4}{*}{105382} & HR4618 & 4.4 & B6IIIp He-weak & 53011.195 & $-923 \pm 86$ & 751 & 105.6 \\
\hline & & & & 53015.247 & $-431 \pm 109$ & & \\
\hline & & & & 53144.003 & $840 \pm 58$ & & \\
\hline & & & & 53224.989 & $-715 \pm 79$ & & \\
\hline \multirow[t]{5}{*}{131120} & HR5543 & 5.0 & B7p He-weak & 52824.158 & $-228 \pm 110$ & 130 & 2.3 \\
\hline & & & & 53020.353 & $-137 \pm 74$ & & \\
\hline & & & & 53030.366 & $63 \pm 69$ & & \\
\hline & & & & 53225.027 & $92 \pm 57$ & & \\
\hline & & & & 53234.102 & $-39 \pm 72$ & & \\
\hline \multirow[t]{3}{*}{138769} & HR5781 & 4.5 & B3IVp He-weak & 52904.027 & $-16 \pm 58$ & 225 & 5.9 \\
\hline & & & & 52908.022 & $-260 \pm 84$ & & \\
\hline & & & & 53234.120 & $-289 \pm 101$ & & \\
\hline
\end{tabular}

\section{Observations}

The observations reported here have been carried out at the European Southern Observatory with FORS 1 (FOcal Reducer low dispersion Spectrograph) mounted on the 8-m Melipal telescope of the VLT. This multi-mode instrument is equipped with polarisation analyzing optics comprising super-achromatic half-wave and quarter-wave phase retarder plates, and a Wollaston prism with a beam divergence of $22^{\prime \prime}$ in standard resolution mode. We used the GRISM 600B in the wavelength range 3480-5890 $\AA$ to cover all hydrogen Balmer lines from $\mathrm{H} \beta$ to the Balmer jump. The spectral resolution of the FORS 1 spectra taken with this setting was $R \sim 2000$. The determination of the mean longitudinal fields using FORS 1 is described in detail in Hubrig et al. (2004). While the accuracy of the longitudinal magnetic field determination using metal lines strongly depends on the width of the spectral lines used in the analysis, the advantage of using FORS 1 is especially obvious in fast rotating stars with large $v \sin i$ values where for the measurement of polarisation exclusively intrinsically broad hydrogen Balmer lines are used.

For each star we usually took four to eight continuous series of two exposures with the retarder waveplate oriented at different angles. The spectropolarimetric capability of the FORS 1 instrument in combination with the large light collecting power of the VLT allows us to achieve a S/N ratio up to a few thousands per pixel in the one-dimensional spectrum, as required to detect low polarisation signatures produced in the spectral lines by longitudinal magnetic fields of the order of hundred Gauss and less.
The mean longitudinal magnetic field is the average over the stellar hemisphere visible at the time of observation of the component of the magnetic field parallel to the line of sight, weighted by the local emergent spectral line intensity. It is diagnosed from the slope of a linear regression of $V / I$ versus the quantity $-g_{\text {eff }} \Delta \lambda_{z} \lambda^{2} \frac{1}{I} \frac{\mathrm{d} I}{\mathrm{~d} \lambda}\left\langle B_{l}\right\rangle+V_{0} / I_{0}$ (Bagnulo et al. 2002). The study of a large sample of magnetic and non-magnetic Ap and Bp stars already proved that this regression technique is very robust so that stars with detections with $\left\langle B_{l}\right\rangle>3 \sigma$ possess magnetic fields.

Individual magnetic field measurements are given in Table1 In the first four columns we give the HD number, another identifier, the visual magnitude and the spectral type. The modified Julian date of the middle of the exposures and the measured mean longitudinal magnetic field $\left\langle B_{1}\right\rangle$ are presented in Columns 5 and 6 respectively. The rms longitudinal magnetic field is given in Column 7. It is computed from all $n$ measurements according to:

$$
\overline{\left\langle B_{l}\right\rangle}=\left(\frac{1}{n} \sum_{i=1}^{n}\left\langle B_{l}\right\rangle_{i}^{2}\right)^{1 / 2} .
$$

The reduced $\chi^{2}$ for these measurements are presented in Column 8, following:

$$
\chi^{2} / n=\frac{1}{n} \sum_{i=1}^{n}\left(\frac{\left\langle B_{l}\right\rangle_{i}}{\sigma_{i}}\right)^{2}
$$

Magnetic observations of all four stars phased with the rotation periods obtained by Briquet et al. (2004) are presented in Fig. 1] 

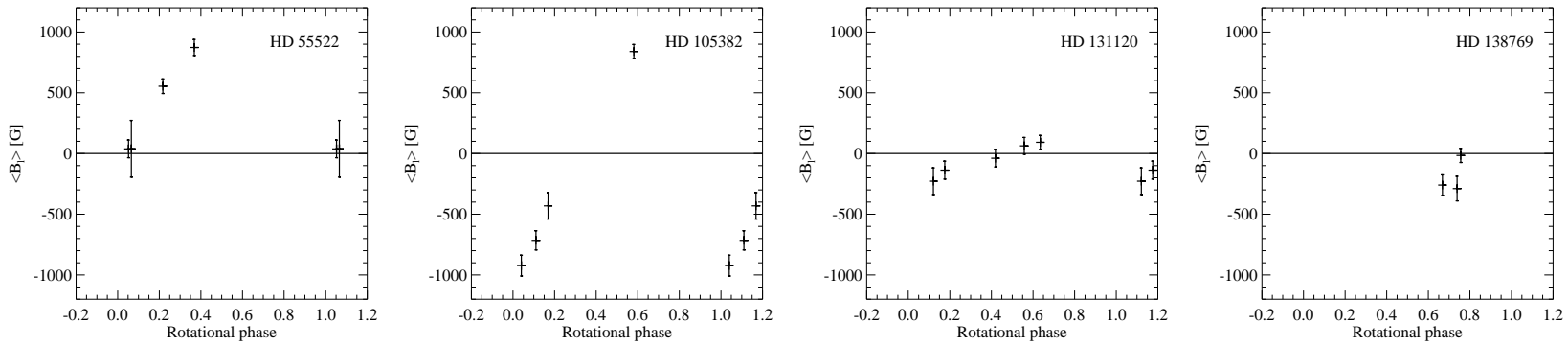

Fig. 1 The mean longitudinal magnetic field variability over the stellar rotation cycle for HD 55522, HD 105382, HD 131120, and HD 138769.

\section{Discussion}

The fundamental parameters of the studied stars are presented in Table 2 The effective temperature $\log \left(T_{\text {eff }}\right)$ and the surface gravity $\log (g)$ (Columns 2 and 3 in Table 2) were derived in Briquet et al. (2004). To determine other stellar parameters, a grid of main-sequence models has been used, which was calculated with the Code Liégeois d'Évolution Stellaire (version 18.2, written by R. Scuflaire), assuming solar composition. For a detailed description, see "grid 2" in De Cat et al. (2006). The mass $M$, the radius $R$, the luminosity $\log \left(L / L_{\odot}\right)$, and the age of the star expressed as a fraction of its total main-sequence life $f$ are presented in Columns 4 to 7 in Table 2 The projected rotational velocity values (Column 8) in Table 2 have been taken from Briquet et al. (2004). To determine the rotational periods (Column 9) presented in the last column of Table 2 Hipparcos photometry, multi-colour Geneva photometry and high-resolution CES spectra have been used.

Our measurements show a clear variability of the mean longitudinal magnetic field over the rotational period on the surface of HD 55522 and HD 105382 (Fig. 11. The abundance maps for He and Si surface distribution obtained by Briquet et al. (2004) for HD 55522 revealed depleted silicon regions along the equator and enhanced silicon regions located close to the rotational poles. Around phase 0.75, a strong helium spot is visible on the equator surrounded by a very depleted region. If we assume that $\left\langle B_{l}\right\rangle$ varies as a sine function with the stellar rotation period, both extrema of the magnetic field would correspond to rotational phases where $\mathrm{He}$ and $\mathrm{Si}$ are found depleted.

The maps for HD 105382 showed two strongly overabundant $\mathrm{He}$ spots just above the equator, which are visible around phases 0.08 and 0.67 . A less strong overabundant third $\mathrm{He}$ spot became visible at phase 0.5. The regions with depleted helium showed enhanced silicon and vice versa. One overabundant He spot (or underabundant Si spot) corresponds to the negative extremum of the measured $\left\langle B_{l}\right\rangle$ while the other overabundant He spot (or underabundant Si spot) coincides with the positive magnetic field extremum, if we assume that the mean longitudinal magnetic field varies sinusoidally with the stellar rotation period.

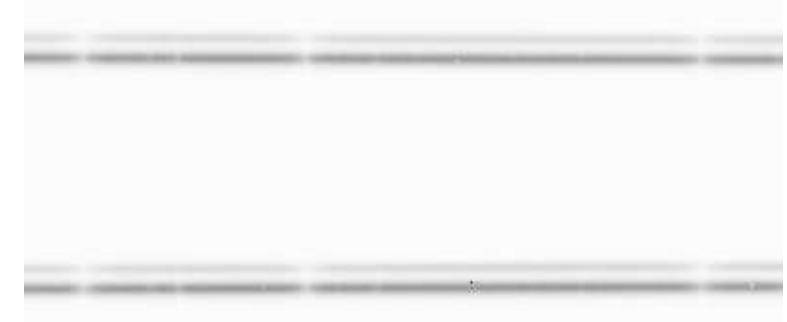

Fig. 2 FORS 1 spectra of HD 138769 taken in right- and left-hand circular polarized light from $\mathrm{H} \epsilon$ to $\mathrm{H} \gamma$. The presence of the close visual companion is clearly visible.

HD 131120 showed two overabundant He spots close to the equator, which have been visible in phases 0.2 and 0.5 . One underabundant He spot was visible around the phase 0.75 . The presence of a magnetic field was not confirmed by Borra et al. (1983). Also none of our five magnetic field measurements is significant at $3 \sigma$ level. Since in general the presence of chemical inhomogeneities on the surface of $\mathrm{Ap}$ and $\mathrm{Bp}$ stars is closely connected with the presence of magnetic fields in their atmospheres, we suggest that HD 131120 is likely a very weak magnetic star with a mean longitudinal magnetic field below one hundred Gauss.

For HD 138769, Briquet et al. (2004) found an enhanced Si spot located at the equator which is visible around phase 0.5 whereas a depleted Si region appeared close to the pole. The existence of the polar spot was considered as less secure. As for helium, no abundance distribution could be derived because the deviation between observed and calculated profiles for the best maps was still very large. The star HD 138769 is a close visual double star with a sepa-

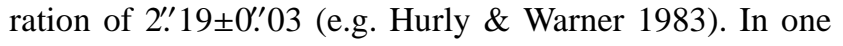
of our FORS 1 spectra we noticed the presence of the close visual component at a separation of $\sim 1$ '"5 (Fig. 2). However, in case of worse seeing the visual components are not separated, and thus the observed spectra are contaminated. Only three magnetic field measurements have been obtained with FORS 1, and among them only the measurement at rotational phase 0.67 is formally significant at $3 \sigma$ level. 
Table 2 Fundamental parameters for the objects in our sample. In the first column we give the HD number. In the following five columns we list the logarithm of the effective temperature, the logarithm of the surface gravity, mass, stellar radius, and the logarithm of the stellar luminosity. The final three columns give the fraction of the main sequence lifetime for each individual star, its $v \sin i$ and the rotational period.

\begin{tabular}{rcccccccc}
\hline \multicolumn{1}{c}{ HD } & $\log \left(T_{\text {eff }}\right)$ & $\log (g)$ & $M / M_{\odot}$ & $R / R_{\odot}$ & $\log \left(L / L_{\odot}\right)$ & $\mathrm{f}$ & $\begin{array}{c}v \sin i \\
{[\mathrm{~km} / \mathrm{s}]}\end{array}$ & $\begin{array}{c}\mathrm{P}_{\text {rot }} \\
{[\mathrm{d}]}\end{array}$ \\
\hline 55522 & $4.241 \pm 0.020$ & $4.15 \pm 0.20$ & $5.5 \pm 0.9$ & $3.3 \pm 1.1$ & $3.0 \pm 0.3$ & $0.46 \pm 0.33$ & $70 \pm 5$ & $2.729 \pm 0.001$ \\
105382 & $4.241 \pm 0.020$ & $4.18 \pm 0.20$ & $5.4 \pm 0.9$ & $3.3 \pm 1.0$ & $2.9 \pm 0.3$ & $0.43 \pm 0.32$ & $75 \pm 5$ & $1.295 \pm 0.001$ \\
131120 & $4.261 \pm 0.020$ & $4.10 \pm 0.20$ & $6.1 \pm 1.1$ & $3.7 \pm 1.2$ & $3.1 \pm 0.3$ & $0.53 \pm 0.30$ & $86 \pm 5$ & $1.569 \pm 0.001$ \\
138769 & $4.243 \pm 0.020$ & $4.22 \pm 0.20$ & $5.4 \pm 0.8$ & $3.2 \pm 0.8$ & $2.9 \pm 0.3$ & $0.38 \pm 0.30$ & $85 \pm 5$ & $2.089 \pm 0.001$ \\
\hline
\end{tabular}

\section{Conclusions}

In summary, we conclude that the magnetic fields are clearly detected in HD 55522 and HD 105382, whereas the presence of a magnetic field in HD 131120 and HD 138769 still needs confirmation by more accurate spectropolarimetric observations. Since the measured longitudinal magnetic fields in HD 55522 and HD 105382 are rather strong, these stars seem to be excellent candidates to study their magnetic field geometry based on a larger number of magnetic field measurements.

The position of the four studied He variable Bp stars in the H-R diagram is shown in Fig. 3 In the same diagram we also present the distribution of SPB and $\beta$ Cephei stars with studied magnetic fields (Hubrig et al. 2006a; Hubrig et al. 2006b; Neiner et al. 2003a; Neiner et al. 2003b; Donati et al. 2001). Filled circles and squares correspond to the pulsating stars with detected magnetic fields. Non-pulsating chemically peculiar hot $\mathrm{Bp}$ stars and pulsating stars evidently coexist in the SPB and $\beta$ Cephei instability strips. It is especially intriguing that the magnetic fields of hot Bp stars either do not show any detectable variations or vary with periods close to one day, which is of the order of the pulsation and rotation period range of SPB stars (Bohlender et al. 1987; Matthews \& Bohlender 1991). The measured magnetic fields in 14 SPB stars and in 3 stars of $\beta$ Cephei type, however, demonstrate that their fields are rather weak in comparison to the $\mathrm{kG}$ fields detected in magnetic Bp stars. This seems to indicate that very strong magnetic fields are not co-existent with oscillations, or stars with stronger magnetic fields have much lower pulsation amplitudes. Such an observational evidence has recently been mentioned for rapidly oscillating stars by Kurtz et al. (2006). We note however that the pulsation mechanism is very different in the two groups of stars.

Clearly, the presently available observational results are still marginal. Further studies of magnetic fields in hot B stars, both pulsating and non-pulsating, are necessary to provide important information on the magnetic field geometry in these stars to test theoretical predictions related to the origin of magnetic fields (e.g., Mullan \& MacDonald 2005; Spruit 2002).

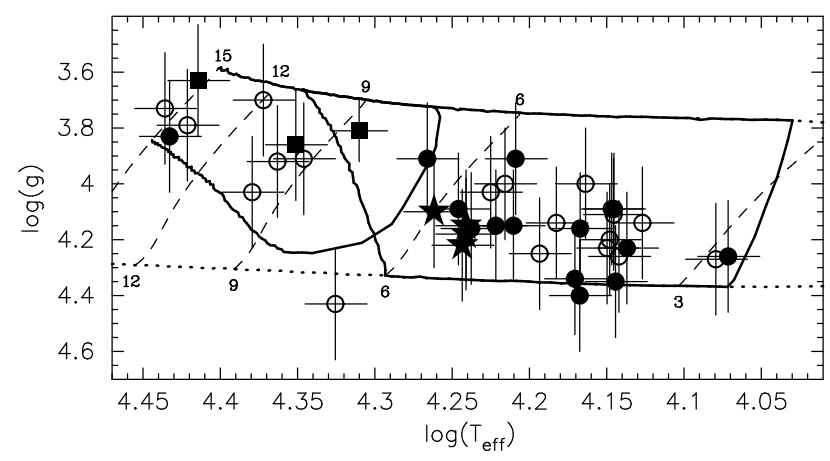

Fig. 3 The position of the four studied Bp stars in the $\mathrm{H}-\mathrm{R}$ diagram is indicated by stars. We also show by circles the position of $\beta$ Cephei and SPB stars for which Hubrig et al. (2006a) searched for the presence of a magnetic field. In addition we represent by squares the three magnetic Btype pulsators discovered by Neiner et al. (2003a,b) and Donati et al. (2001). Filled circles and squares correspond to stars with detected magnetic fields. The full lines represent the boundary of the theoretical instability strips for modes with a frequency between 0.2 and $30 \mathrm{~d}^{-1}$ and $\ell \leq 3 \mathrm{com}$ puted for the main sequence models with $M \in[2,15] M_{\odot}$ in "grid 2" of De Cat et al. (2006) for which Z=0.015, X=0.71, $\alpha_{\text {conv }}=1.75, \alpha_{\text {over }}=0.0$, and the standard metal mixture of Asplund et al. (2005). The lower and upper dotted lines show the ZAMS and TAMS, respectively. The dashed lines denote evolution tracks for stars with $M=15,12,9,6$, and $3 M_{\odot}$.

\section{References}

Aerts, C., De Cat, P., Peeters, E., et al., 1999, A\&A, 343, 872 Asplund, M., Grevesse, N., \& Sauval, A.J., 2005, in ASP Conf. Ser. Vol. 336, Cosmic Abundances as Records of Stellar Evolution and Nucleosynthesis, eds. T.G. Barnes III \& F.N. Bash, 25

Bagnulo, S., Szeifert, T., Wade, G.A., et al. 2002, A\&A, 389, 191 Bohlender, D.A., Landstreet, J.D., Brown, D.N., \& Thompson, I.B., 1987, ApJ, 323, 325

Borra, E.F., Landstreet, J.D., Thompson, I., 1983, ApJS, 53, 151

Briquet, M., Aerts, C., Lüftinger, T., et al., 2004, A\&A, 413, 273

De Cat, P., Briquet, M., Aerts, C., et al., 2006, CoAst, 147, 48

Donati, J.-F., Wade, G.A., Babel, J., et al., 2001, MNRAS, 326, 1265 
Hubrig, S., Briquet, M., Schöller, M., et al., 2006a, MNRAS, 369, 61

Hubrig, S., North P., Schöller, M., Mathys, G. 2006b, AN, 327, 289

Hubrig, S., Szeifert, T., Schöller, M., et al., 2004, A\&A, 415, 661

Hurly \& Warner, 1983, MNRAS, 202, 761

Kurtz, D.W., Elkin, V.G., Cuhna, M.S. et al., submitted to MNRAS

Mathias, P., Aerts, C., Briquet M., et al., 2001, A\&A, 379, 905

Matthews, J.M., \& Bohlender, D.A., 1991, A\&A, 243, 148

Michaud, G., 1970, ApJ, 160, 641

Michaud, G., Megessier, C., \& Charland, Y., 1981, A\&A, 103, 244

Mullan, D.J., MacDonald, James, 2005, MNRAS, 356, 1139

Neiner, C., Geers, V.C., Henrichs, H.F., et al., 2003a, A\&A, 406, 1019

Neiner, C., Henrichs, H.F., Floquet, M., et al., 2003b, A\&A, 411, 565

Spruit, H.C., 2002, A\&A, 381, 923 